\title{
Research on the Integration of Information Systems Based on BPM
}

\author{
Zhaoxia Gou \\ Huai Insitute of Technology \\ Cangwu Road \#59,222005 \\ Lianyungang Jiangsu China \\ Gouzx@hhit.edu.cn
}

\author{
Xiaoming Liu \\ Huai Insitute of Technology \\ Cangwu Road \#59,222005 \\ Lianyungang Jiangsu China \\ Liuxm029@gmail.com
}

\begin{abstract}
The proliferations of isolated information management systems in colleges raise many issues of system integration, data sharing etc. In this paper, we describe the current problems in the information construct and analyze the benefits and limitations of platform integration options we then discuss how BPM works as an integration tool, and show its benefits.
\end{abstract}

Keywords- information constructs; integration; BPM

\section{INTRODUCTION}

With the development of computer power, networking and the Internet, more and more applications and information management systems are being used in colleges for faculty and students' services. They are also playing an increasing role in college business management. However, the coming of new technologies like the Internet of Thing, Clouding computing and big data highlights the unsuitability of traditional information management systems to meet new requirements, especially for the fresh needs of data mining. The government now pays more attention to information constructs and applications, given its investment of $4 \%$ of GDP for education.

The higher educational information construct has experienced networking, digitization, and the new information and intelligence age. It has also had huge changes in the business units and data requirements this has resulted in isolated information management systems that have to be updated to adapt to the new situation. One way to adapt is to discard the past systems and establish new management systems. However, for financial management controls, this is not practical for most colleges. Another way is to integrate the past systems with newer ones, and make use of them fully. When the financial reporting or the past systems cannot meet new requirements then these legacy systems can be discarded [1]. In this paper we discuss how to integrate the past information systems with the latest systems, and through this integration, how to achieve data sharing, interconnection and cooperation based on business procession management (BPM).

\section{THE CURRENT PROBLEM IN INFORMATION CONSTRUCT}

There are many colleges in China. Every college is in a different stage of information construct and each has a variety of computer applications. However, for most of them there are the following common issues:
1) The management information constructs, and operation mechanism need to be perfected. Every section and branch has a unique mission in the school's business management and the information development tasks and management responsibilities are not clear and definite. They satisfy their information requirements for their own needs, and never care about data sharing with others. There is an information support department in the college, but it is just for the implementation and support of the section's computer applications, and has no any other right to limit this phenomena. Thus, it needs a management scheme to organize and manage the information construct from the top to the bottom.

2) Lack of scientific planning. In the information construct of the whole school, there is insufficient comprehension and grasp of the about current information situation, and about the actual situation of the school, so it is difficult to effectively guide the implementation of the information construct work [2].

3) Shortage of system integration. Due to the rapid growth of information technology and the limitations of school development objective conditions, many $\mathrm{C} / \mathrm{S}$ or $\mathrm{B} / \mathrm{S}$ are running at the same time. Thus, data sharing is inefficient between the systems. Wide differences in data standards -"information isolated island" issues --are very important and system interfaces are underdeveloped [3].

4) Poor scalability and business flexibility. Various business systems were distributed independently and fragmentally, and data and applications could not provide for resource sharing, and expand to meet the needs of new and versatile business applications. These systems were unable to achieve unified control and maintenance, or to ensure application systems of virus protection, network security, data security, etc.

There are other many problems happening in the information systems. The more important problems, however, are in the information construct, and data standardization, storage, sharing, accumulation, and analysis. The application of business systems also needs to be more user-friendly and to offer an excellent user experience [4].

\section{THE CURRENT PlatFORM INTEGRATION AND ISSUE}

The most important ways of integration for the information systems are to develop unified identity authentication, a unified information portal and public data centers to support the integration of data, authentication, the portal and business. 


\section{A. The Current Platform Integration}

Unified identity authentication platforms provide functions to manage multiple application systems and identity authentication, improves the level of application system user management, and reduces system authority management chaos. It also uncovers hard to find hidden issues. By using this system, users need not memorize different passwords and identities, for the business system provides a consistent access service model, through the information portal platform, to realize single sign-on (SSO) and to avoid duplication of investment as a whole.

For all faculty, support staff, scientific research personnel, administrative personnel, students and alumni, it provides centralized management inside a variety of information resources, including news, announcements, notifications, documents, multimedia courseware and application services, including information collection, information query, information release, etc. The portal platform integrates the information of the digital campus with the application of resources through a unified web page. The user, as long as he or she has an account, can access all resources within the scope of authority. To adopt a flexible access control mechanism based on its own set of information resources classification and user access, the content and forms of information can be customized. Users thus can enjoy the individualized information service provided; personalized information services.

The public data center is the campus basic data sharing platform. The data of various business units is centralized and management unified, to ensure the authority and accuracy of data for each business application system and for all kinds of service systems. This provides authoritative and accurate data support, absorbs the increasing volume of data in the digital campus, and ensures exchange of information, processing, and switching results [5].

\section{B. Integration ways}

Data integration is based on the business integration shared data format exchange, the specified data format between different application systems, through the selected communications as per the medium (for example, file sharing exchange, FTP file exchange, exchange database table, based on the TCP/IP socket communication protocol, message queue, etc.). The program automatically or manually performs data integration goals.

Certification Integration is an integrated model based on the unified identity authentication platform for system users and permissions consolidation. It provides user permissions unified management and unified certification, and audit systems to access the unified identity authentication platform, thus achieving real 4A safety standards.

Portal Integration is through the IFRAME, Web Clipping, SSO, comprehensive data display, links, RSS feeds and other mechanisms, to integrate each departments' web site, data centre, and internet news information into the unified information portal platform

Business Integration is the most complex, but it is the most valuable type of integration, to resolve the form of application amongst several departments environment using business logic. In such case, the portal application data (reply) will immediately affect multiple position information or many steps of the defined information or processing workflows. The new student registration, for example, can affect the data in multiple systems, such as the library management system and IC card system, through the business integration in the portal; it greatly reduces the data risk of inconsistency on the workflow/business logic services and processes [8].

\section{The potential issues}

The integration of the platforms is all based on SOA (service-oriented architecture), and encapsulate the systems to the services, but those services still have limitations:

1) The independent business operation in different information management systems. The above platform which we just mentioned still runs as its own web application. The unified information portal shows the main information distribution pages for the web users and it is easy and convenient to access the different information management systems. It does not waste time to remember the websites and opens the main pages of the different information management systems, but it has the underlying problem that the operational mission and related workings are still locate in the independent unit's information management system. The business tasks still are independent from the unified information portal, and work as isolated systems.

2) The data centre reduces problems of data sharing and consistency. In college business applications, there are many kinds of data format. Like fundamental datum. This data is from essential information about the faculty, students, the teaching equipment, real estate etc. This data is static but as those entities change, they generate much more data derived from the entity motion or operational results. This evolving data belongs to the management system itself and is shifted to the data centre. Thus, problems about data writing rights and data cleaning, whether the data changes under the operation or not, does not affect the data security and consistence.

\section{INTEGRATION BASED ON BPM}

\section{A. Business Process Management}

BPM has been referred to as a "holistic management" approach to align an organization's business processes with the wants and needs of clients [7]. BPM uses a systematic approach in an attempt to continuously improve business effectiveness and efficiency while striving for innovation, flexibility, and integration with technology. It can therefore be described as a "process optimization process". It is argued that BPM enables organizations to be more efficient, more effective and more capable of change than a functionally focused, traditional hierarchical management approach [8].These processes can impact the cost and revenue generation of an organization. As a managerial approach, BPM sees processes as strategic assets of an organization that must be understood, managed, and improved to deliver value-added products and services to clients. BPM closely resembles other total quality management or continuous 
improvement process methodologies or approaches. BPM, however, goes a step further by stating that this approach can be supported, or enabled, through technology to ensure the viability of the managerial approach in times of stress and change. In fact, BPM offers an approach to integrate an organizational "change capability" that is both human and technological. As such, many BPM articles and pundits often discuss BPM from one of two viewpoints: people and/or technology.

Process management platforms can provide visual process designing, debugging, and graphical state tracking. They can automatically generate file format conversion of process definitions, etc. At the same time, they can provide process management tools to query process instances execution status, to perform some privileged operation process instances (e.g., terminate, suspend, recall, etc.), and to process instance data management, online adjustment process definition and so on. With the process mapping and processing configuration process administrator, the terminal user queries the current state of the process circulation, and sees displays with all the flow diagram. Users can also design and configure the process flow through the browser. Meanwhile the system users, permissions, interface, and other settings can also be conveniently configured through the browser.

1) Process design and management

BPM Intuitively shows the process doing work, initiating the process and handling process, including process working time, reminders and warnings, and important or common processes that can be customized to facilitate quick launch and tracking statistics; it is graphical process modeling based on the Web, and can copy, import and export the process template XML format, conditional judgments, sub processes, and programmable system automatic working links.

2) Support all kinds of business form sheets

The BMP supports various component libraries, Microsoft office and many kinds of the definitional text forms. It integrates related data links and operations inside and outside of the system.

3) Process transaction

The workspace provides extensive option for decision approval, and decision approval comments It supports multilevel and multiple signing, entrusted handling, consultation and other complex decision-making needs; Form sheets are recorded and displayed graphically in tree structures, using different colors to deal with, and illustrate the flow path and the current state of the transaction, Flow charts strikingly tag the actual path, thoroughly maintaining a record of process flow works and operation history.

4) Process monitoring and maintaining

The visualized process and flow chart monitoring, can directly display the approving officer, the business opinions and processing status. The user can revise the sequence, node status, and operational officer in the procession and prompt the mission to the operational officer to make sure the process goes smoothly.

5) Process integration and analysis

The BPM adopts an event driven mechanism, and can trigger events to start the interaction of the process data It supports XML Web Services, Java processes or database SP store processes; which can be configured for data fusion and transmission between the implementation processes and subprocesses. BPM can configure or program nodes to work automatically so that when the data flow comes to the node, the system automatically performs stipulated tasks in the operation, and triggers email, fax, and other systems for complex data interaction. After the completion of the process, it can generate the requested reports, documents, or other needed papers, and make a graphical model to show a profuse data table to support the decision reference. In addition, BMP can provide more analysis of every step or the whole process by duration, and clearly get the total amount of every data flow transaction, including the participants, average consumed time, and the efficiency of every approval stage, thus providing the basis for performance appraisal and process optimization.

\section{B. The Benefits of BMP}

As we have discussed in this paper, BMP can resolve the following problems:

1) Integrate the distributed business process. For the isolated management systems, BPM assembles together every management system's business process and displays the full transactions to the terminal users; everyone selects their own requirements using BPM services.

2) Integrate the triggered data. When the BMP runs, the operation and order trigger the corresponding management systems and generate fresh data. This data will be helpful for data warehousing and performance assessment in the future.

3) Integration of management systems, applications, data and business. Concrete management systems are to provide services and user will try hard to use them to collect data or forms from many applications. When BMP runs to meet user requirements, the process will provide business services, decision-making services, raw data and analysis. These working procedures and dynamic motion records will be loaded in the servers, creating new big data.

\section{SUMMARIES}

Although BPM had used in enterprise management for a long time, there is no particular example to follow in its use for information integration on the campus, some colleges use process management to deal with the business transactions, but this is strictly operational, and not related to data, tracking and other sophisticated IT requirements.

There are important reasons for Chinese colleges to adopt BPM in IT management. . The college in China is diverse; different department or branches have self-governing rights and responsibilities. In addition, some information management systems must also report to upper government. Interface opening and data sharing is difficult and complex, but BPM holds promise as a tool that can be used by colleges to meet the future challenges faced by information management systems in colleges 


\section{ACKNOWLEDGMENT}

This research was supported by China National Foundation NO.61103017, Lianyungang Science and Technology Project No.CG1215, Lianyungang Social Development Project No.SH1212 and Z2012131

\section{REFERENCES}

[1] Shen Lining. Research on Uncertainties in Enterprise Information System Integration. INFORMATION STUDIES:THEORY \& APPLICATION 2008.31(1):120-126.

[2] Wang Lipeng, Chen Ya,etc., The Construction of Digital Campus in Chinese Universities and Its Speculation INFORMATION SCIENCE2004.22(3):356-362.

[3] Xu xin, Su Xinning Building a New Generation Digital Campus of University New Technology of Library and Information Service 2008(5):48-54.

[4] Yan Shuting, Liu Jiaxin. Research of External Information Sharing Mechanism of University Scientific Research Management System Science and Technology Management Research 2013.12:85-90.

[5] Vom Brocke, J.HKVJH \& Rosemann, M. (2010), Handbook on Business Process Management: Strategic Alignment, Governance, People and Culture (International Handbooks on Information Systems) (Vol. 1). Berlin: Springer.

[6] Ryan K. L. Ko. A computer scientist's introductory guide to business process management (BPM), ACM Press Crossroads2009 .15(4).

[7] Li Zhonglin. The problems and countermeasures based on university digital campus construction. Vocational Technical Education Forum, 2012, (26):49-50.

[8] Liu X. M.,Jia C.Y. The Analysis and Research on Digital Campus Construction Model. ITME 2013. LNEE. 269: 2195 - 2199. 
